\title{
Conciencia emocional en la práctica formativa del profesorado de educación física
}

\author{
FELIPE NICOLÁS MUJICA JOHNSON* \\ Universidad Católica de Temuco - Chile \\ NELLY DEL CARMEN ORELLANA ARDUIZ \\ Universidad de Playa Ancha de Ciencias de la Educación - Chile \\ Recibido el 10-06-18; primera evaluación el 15-08-19; \\ segunda evaluación el 16-08-19; tercera evaluación el \\ 17-08-19; aceptado el 20-08-19
}

\section{RESUMEN}

Se ha realizado un estudio para identificar la percepción y atribución emocional de los estudiantes de Pedagogía en Educación Física durante su práctica educativa. La metodología responde al enfoque cualitativo. Los participantes son 22 estudiantes que cursan su tercer año académico. Los datos fueron recopilados a través de un diario personal y fueron sometidos a un análisis inductivo de contenido, utilizando el programa Atlas.ti 7.5. Los resultados manifiestan que los participantes perciben mayoritariamente emociones positivas para su bienestar subjetivo, mientras que de las atribuciones emergieron dos categorías centrales, nombradas interacción social y rol pedagógico. Se concluye que las emociones emergen principalmente por el deseo de querer lograr una adecuada práctica pedagógica, el afecto recibido y la contribución en el aprendizaje del alumnado escolar

Palabras clave: educación superior; relaciones sociales; formación de profesores, investigación educativa, emociones.

\footnotetext{
* Licenciado en Educación. Profesor de Educación Física y magíster en Ciencias de la Actividad Física y del Deporte de la Universidad de Playa Ancha de Ciencias de la Educación (UPLA). Docente de Pedagogía en Educación Física - UPLA. Doctorando del programa de Doctorado en Ciencias de la Actividad Física y del Deporte de la Universidad Politécnica de Madrid. Docente-investigador del Centro de Investigación Escolar y Desarrollo (CIED) de la Universidad Católica de Temuco. Correo electrónico: fmujica@live.cl

** Profesora de Estado en Educación Física de la Universidad de Chile. Magíster en Educación Física de Escuela Superior de Deporte de Alemania. Doctora en Filosofía y Educación de la Universidad Nacional a Distancia de España. Doctora en Filosofía de Leibniz Universität de Alemania. Docente de Pedagogía en Educación Física y docente del Magíster de Ciencias de la Actividad y del Deporte de la Universidad de Playa Ancha de Ciencias de la Educación. Correo electrónico: norellan@upla.cl
} 


\section{Emotional awareness in the formative practice of physical education teachers}

\section{Abstract}

A study has been carried out to identify the perception and emotional attribution of the students of Pedagogy in Physical Education during their educational practice. The methodology responds to the qualitative approach. The participants are 22 students who are in their third academic year. The data was collected through a personal diary and subjected to an inductive content analysis, using the Atlas.ti 7.5 program. The results indicate that the participants mostly perceive positive emotions for their subjective wellbeing, while the attributions emerged two central categories, named social interaction and pedagogical role. It is concluded that emotions emerge mainly due to the desire to achieve an adequate pedagogical practice, the affection received and the contribution in the learning of schoolchildren.

Keywords: higher education; social relationships; teacher training, educational research, emotions.

\section{Consciência emocional na prática formativa de professores de educaçáo física}

\section{Resumo}

Um estudo foi realizado para identificar a percepção e atribuição emocional dos alunos de Pedagogia em Educaçâo Física durante sua prática educativa. A metodologia responde à abordagem qualitativa. Os participantes são 22 estudantes que estâo em seu terceiro ano acadêmico. Os dados foram coletados através de um diário pessoal e submetidos a uma análise de conteúdo indutivo, utilizando o programa Atlas.ti 7.5. Os resultados indicam que os participantes percebem principalmente emoçóes positivas para o seu bem-estar subjetivo, enquanto as atribuições emergiram duas categorias centrais, denominadas interação social e papel pedagógico. Conclui-se que as emoçôes emergem principalmente devido ao desejo de alcançar uma prática pedagógica adequada, o afeto recebido e a contribuição na aprendizagem dos escolares.

Palavras-chave: Educação superior; relaçôes sociais; formação de professores, pesquisa educacional, emoçôes. 


\section{INTRODUCCIÓN}

En Chile, se han establecido estándares educativos que fomentan el desarrollo integral del estudiante en la formación docente de Educación Física (EF), mencionando explícitamente la dimensión afectiva (Ministerio de Educación, 2014). Esta ha sido conceptualizada por este mismo organismo como «un conjunto de emociones, estados de ánimo y sentimientos que permean los actos de las personas, incidiendo en el pensamiento, la conducta y la forma de relacionarse con uno mismo y con los demás» (Ministerio de Educación, 2013, p. 6). Esta idea pedagógica reconoce que el ámbito emocional cumple un rol relevante en el espacio educativo, las cuales son vivenciadas desde el ingreso a la universidad hasta que se recibe el diploma de titulación, aunque también se ha identificado la implicación que tienen los recuerdos con contenido emocional previos a la universidad, en algunas construcciones cognitivas relacionadas con la profesión, como es el caso de la vocación docente (Mujica y Orellana, 2016). Incluir esta área en la labor pedagógica es una tarea compleja, ya que las instituciones académicas formales del país han ocupado una posición periférica en relación con el tema (Moreno, Trigueros y Rivera, 2013), por lo que es difícil entender cuál puede ser su importancia dentro de las aulas de clases o de las actividades que requieren un desempeño más práctico.

Entre las emociones que en el contexto educativo han sido clasificadas con un valor positivo se encuentra la alegría, la seguridad o la confianza, ya que favorecen el aprendizaje, mientras que, por el contrario, las emociones con un valor negativo como el miedo, la tristeza o la ira lo dificultarían (Um, Plass, Hayward y Homer, 2012; López, 2016). No obstante, aunque se han realizado relaciones «entre emociones y aprendizaje, la clasificación estandarizada de positivas y negativas ha sido criticada por su simplicidad, señalándose que si bien hay evidencia que coincide con este planteamiento, la función de las emociones tiene un mayor matiz en el aprendizaje» (Mujica, Inostroza y Orellana, 2018c, p. 116). Asimismo, Vogel y Schwabe (2016) sostienen que el estrés de bajo nivel permite favorecer el proceso de enseñanza-aprendizaje, debido a que niveles medios y altos lo afectarían negativamente. En este sentido, tanto las emociones positivas como las negativas podrían favorecer el acto educativo (Mujica et al., 2018c; Tyng, Amin, Saad y Malik, 2017). Esta misma clasificación se encuentra vinculada al bienestar o malestar subjetivo de las personas (Rodríguez-Fernández y Goñi-Grandmontagne, 2011), la cual ha sido utilizada en una propuesta teórico-práctica de educación emocional (Bisquerra et al., 2016), estableciendo que «la finalidad de esta educación es el bienestar, teniendo presente que su construcción incluye la regulación de las emociones negativas y la potenciación de las positivas» (Mujica, Orellana y Canepa, 2018, p. 9). 


\section{Marco teórico}

Esta forma de valorar las emociones, en el contexto pedagógico, no ha estado exenta de críticas, debido a que se reclama una perspectiva que considere el marco ético de la educación emocional (Marina, 2005), ya que de lo contrario el proceso educativo estaría siendo guiado por conocimiento psicológico y no conocimiento pedagógico (Prieto, 2018). Este enfoque educativo promueve la formación en competencias emocionales, durante la formación docente, para que los estudiantes puedan afrontar con éxito la compleja tarea educativa y de esta forma prevenir desequilibrios en la salud psicológica del profesorado, como también favorecer su formación pedagógica (Bisquerra, 2005; Palomera, Ferández-Barrocal y Brackett, 2008). Estos planteamientos son coherentes y adecuados con la realidad que vivencia el profesorado, caracterizada por altos niveles de estrés laboral, por lo que pueden beneficiarse de este tipo de estrategias de afrontamiento, evitando así las crisis sobre su actitud laboral y su salud mental (Vogel y Schwabe, 2016). Entre las competencias emocionales que establece la propuesta mencionada de educación emocional, se encuentra la percepción o conciencia emocional, la comprensión de las emociones y la regulación emocional que se destaca por ser la más difícil de lograr, las cuales se enfocan en el alumnado escolar como en su profesorado (Bisquerra, 2005; Bisquerra et al., 2016; Koole, 2009). A partir del desarrollo de dichas competencias, se espera que los futuros docentes puedan «establecer relaciones interpersonales de buena calidad o lograr un ajuste emocional en las situaciones conflictivas que acontecen antes, durante o después de la gestión de la clase» (Mujica, 2018a, p. 66).

En este estudio, se abordará la conciencia emocional de futuros maestros que realizan su práctica pedagógica en un centro educativo escolar, considerando que dicha competencia es la base de las otras competencias mencionadas (SamperGarcía, Mesurado, Richaud y Llorca, 2016). Precisamente, esta competencia ha sido conceptualizada como un proceso atencional que se encuentra interconectado con algunas funciones interpretativas y evaluativas, permitiendo a las personas percibir e interpretar de forma cualitativa sus emociones con el fin de ubicar sus causas (Oosterveld, Miers, Merum y Ly, 2008). A través de estos antecedentes, es posible comprender que la implicación de las emociones en el ámbito educativo se encuentra en un proceso de desarrollo, pero existe la certeza de que se encuentran integradas con los procesos cognitivos (Lazarus, 2000; Maturana, 2001; González, 2009; Cabezas, 2013; Salmurri, 2015; Mora, 2017), de modo que «la afectividad y la cognición son inseparables, el mismo Piaget ve las relaciones entre inteligencia y afectividad» (Del Valle, 
1998, p. 171). Asimismo, Mora (2017) señala que las emociones son las encargadas de activar como de mantener la atención y la curiosidad, de modo que, respecto al proceso educativo, manifiesta que «son la base más importante sobre la que se sustentan todos los procesos de aprendizaje y memoria» (p. 66).

\subsection{Atribución causal de las emociones}

Cada vez que una persona experimenta una emoción, tiene la posibilidad de analizar su origen y atribuirla a estímulos que la hayan activado, los cuales responden al interrogante de por qué se piensa que se ha sentido esa emoción en un momento determinado, de modo que una atribución consiste siempre en una inferencia, así como percibir significa, en todos los casos, una construcción mental (Crespo y Célio, 2014). Por consiguiente, una atribución representa la certeza de los significados que se encuentran asociados a un hecho, de modo que los procesos de atribución de las personas descubren las causas de los sucesos o los comportamientos (Sanz, Menéndez, Rivero y Conde, 2017). Este concepto fue desarrollado por el psicólogo austriaco Fritz Heider, quien en el año 1958 inició una línea de investigación orientada a explicar el fenómeno que induce a las personas a indagar en las causas de los hechos percibidos, partiendo de la idea de que toda persona tiene un saber sobre sí misma y los demás (Crespo, 1982). Por esta razón, este investigador señala que las personas se inclinan a creer que los sucesos o los comportamientos han sido explicados cuando descubren sus causas que los habrían producido (Sanz, et al., 2017).

En este contexto, la atribución causal se plantea como un proceso fundamental en la percepción y comprensión de la acción humana (Crespo, 1982), las que años más tarde fue ampliada por el psicólogo cognitivo Bernard Weiner, quien construye una teoría sobre la relación entre la atribución causal y la motivación, considerando que la conducta de logro de una persona se produce en función de las atribuciones de éxito o fracaso que se hayan realizado en el pasado, produciendo respuestas afectivas y cognoscitivas que afectarán las futuras conductas de logro. Estas aplicaciones teóricas se han transferido al plano pedagógico, señalándose que las atribuciones causales que realice el alumnado en la etapa secundaria tendrán un peso específico en su desarrollo personal y también dependiendo de ellas, su motivación puede aumentar o disminuir. Por lo tanto, el profesorado debería tener especial cuidado ante el tema y actuar con paciencia al momento de modificarlas cuando exista esta necesidad, debido a que es una tarea que requiere tiempo, debiendo seleccionar las actividades o tareas que se encuentran encaminadas a proporcionar el éxito de los estudiantes, pero sin ser conseguido con excesivas dosis de esfuerzo (Lago, Presa, Pérez y Muñiz, 2003). 
En cuanto a los antecedentes de estudios que hayan abordado esta temática en un contexto educativo similar, es preciso referirse a algunas investigaciones que se han desarrollado en el continente europeo. Cabe destacar que estos estudios cuentan con la participación de estudiantes que cursan la licenciatura de actividad física y deporte, los cuales pueden habilitarse para desempeñarse como docentes de Educación Física en los centros educativos. Así, se mencionará un primer estudio realizado en dos universidades de España y una de Portugal, el cual tuvo por objetivo indagar en las emociones que suscitan los juegos motores con y sin victoria. En conclusión, los autores señalan que los juegos motores son adecuados para educar en competencias emocionales, destacando los juegos cooperativos (sin victoria) como propicios para suscitar emociones positivas para el bienestar subjetivo (Lavega, Aráujo y Jaqueira, 2013). Esta relación entre prácticas motrices y emociones positivas en estudiantes universitarios del ámbito de actividad física y deporte se ha confirmado también en posteriores investigaciones, de modo que se presenta como un importante factor que puede predecir el bienestar subjetivo (Romero-Martín, Gelpi, Mateu y Lavega, 2017; Rovira, López, Lagardera, Lavega y March, 2014). Además del bienestar subjetivo, otro estudio realizado en una universidad española logró asociar desde la perspectiva del alumnado universitario las emociones al ámbito moral, en concreto, al valor de la solidaridad (MujicaJohnson y Jiménez, 2019), lo cual amplía la interpretación en torno a la conciencia emocional.

En América del Sur y, en concreto, en el contexto chileno, también se han identificado algunos estudios sobre las emociones en la formación de docentes en el área de EF. Precisamente, estas investigaciones se han centrado en comprender las emociones desde la perspectiva del estudiantado, haciendo énfasis en los aspectos didácticos que influyen al momento de presenciar y gestionar una sesión de EF (González, Rivera y Trigueros, 2014; Mujica, Orellana y Marchant, 2015; Mujica, 2018b; Orellana, Mujica y Luis-Pascual, 2015). Además, estos estudios han tenido en cuenta la interacción entre los factores del bienestar subjetivo y la formación moral del alumnado. Por esta razón, una de las investigaciones realizadas en una universidad de la región de Valparaíso indica entre sus conclusiones que «la docencia desempeñada con interacciones comprensivas, alegres y basadas en un enfoque constructivistas y de aprendizaje activo, genera una percepción de emociones positivas en los estudiantes» (Mujica, 2018b, p. 412).

Asimismo, este estudio se vincula a la autopercepción emocional por medio de un proceso deductivo-inductivo, en un contexto formativo para la docencia. Con el fin de avanzar en la comprensión del tema, el objetivo 
del presente estudio es identificar la percepción emocional del alumnado que cursa sus estudios en Pedagogía en EF durante su práctica realizada en centros de educación escolar que se ubican en la región de Valparaíso, Chile. En concreto, se analizarán las atribuciones de las emociones positivas (alegría, seguridad, entusiasmo, satisfacción, tranquilidad y diversión) y negativas (enfado, inseguridad, nerviosismo, tristeza, ansiedad y frustración) para el bienestar subjetivo que los estudiantes universitarios perciben durante el desarrollo de su práctica formativa docente en 22 centros de educación escolar.

\section{Metodología}

Este estudio se enmarca en el paradigma de investigación cualitativa, ya que es un estudio interpretativo, el cual es propicio para comprender desde la perspectiva de los estudiantes o profesores en formación, los significados que se encuentran asociados a las emociones en los contextos pedagógicos (Mujica, 2018b; Orellana, et al., 2015). Así, a través de esta metodología, se logró descubrir las atribuciones emocionales durante la práctica pedagógica universitaria, lo cual también estuvo marcado por un proceso dinámico y flexible desde la perspectiva metodológica.

\subsection{Participantes}

Los estudiantes seleccionados para participar del estudio fueron 22 (9 hombres y 13 mujeres) estudiantes de Pedagogía en EF de la Universidad de Playa Ancha de Ciencias de la Educación, que cursan el quinto semestre académico, en su tercer año de estudios. Los participantes fueron seleccionados de forma intencionada, dada la naturaleza de la investigación (Jordán y Méndez, 2017), considerando su condición de estudiante que realiza su proceso de práctica, debiendo asistir durante 10 semanas a un centro escolar. Cada estudiante asistió a un establecimiento educacional, de modo que en total se incluye la experiencia en 22 centros de educación escolar. Durante las primeras dos semanas tuvieron que cumplir un rol de observador de las sesiones y, en las siguientes semanas, debieron intervenir en una parte de la sesión (inicio, desarrollo o final) en coordinación con el profesorado titular de EF.

\subsection{Recolección de información}

Para la recogida de datos durante el trabajo, se ha utilizado el diario personal (Bogdan y Taylor, 2009, p. 141), orientado a indagar los sucesos en que los participantes hayan percibido emociones durante las sesiones de práctica 
y de esta forma poder establecer atribuciones causales. Este instrumento fue nombrado «diario reflexivo», en el cual el/la estudiante podía reflexionar sobre su práctica al término de cada sesión y, además, debía responder a las siguientes dos preguntas abiertas: (a) ¿Qué emociones sentí hoy en la clase? (b) ¿Por qué he sentido esas emociones? Los estudiantes, por exigencia de la universidad, debían asistir como mínimo dos horas semanales al establecimiento educacional y participar de la asignatura de EF. Previo al proceso de asistir al centro educativo, el alumnado participó en procesos de sensibilización y clarificación sobre el tema, abordando conceptualmente lo que es una emoción, un listado de emociones, ejemplificaciones sobre las diferentes emociones, la capacidad de percibir las propias emociones y algunas de sus clasificaciones. Para las consideraciones éticas del estudio, el alumnado universitario participante respondió un consentimiento informado, tomando conciencia de los objetivos del estudio, como del carácter confidencial y anónimo de los datos.

\subsection{Análisis de datos}

Los datos fueron sometidos a un "análisis de contenido inductivo» (Mujica y Orellana, 2018), el cual es reconocido por su aplicación en la investigación educativa (Osses, Sánchez y Ibañez, 2006), y se inició desde los registros narrativos que realizaron los participantes y continuó con la agrupación semántica de los rasgos significativos que fueron agrupados en función de su semejanza semántica. Las fases del análisis siguieron el orden que plantea Mejía (2011): (a) reducción de datos; (b) análisis descriptivo; e (c) interpretación. Por otra parte, se utilizó el programa de análisis de datos cualitativos denominado "Atlas.ti versión 7.5» (Pérez-López, Morales-Sánchez, Anguera y Hernández-Mendo, 2016), con la finalidad de favorecer «la codificación, relación y teorización de los datos, se utilizó el programa informático Atlas.ti versión 7.5» (Mujica y Orellana, 2018, p. 214). Para la codificación, se tuvo en cuenta un principio planteado por Rapley (2014), el cual manifiesta que el análisis de los datos no se debe realizar una vez finalizada la trascripción de los datos, sino que, por el contrario, de modo que comenzó con la lectura y la transcripción de la información obtenida con los diarios reflexivos.

En total, se obtuvieron las narraciones de 172 clases de EF, siendo eliminadas del análisis 26 narraciones, por no mencionar en forma explícita alguna percepción o atribución emocional, de modo que finalmente se analizaron 146 narrativas sobre las emociones percibidas y su atribución. Para garantizar el rigor metodológico de la investigación, se aplicó una triangulación de datos en el ámbito personal, espacial y temporal (Martínez, 2006), que consiste 
en contrastar la información obtenida en diferentes sujetos, espacios y lugares (Aguilar y Barroso, 2015). Como criterio de inclusión de los códigos en los resultados, se estableció que debían ser fundamentados por mínimo tres personas, tres centros educativos y tres sesiones de EF. Esta triangulación se expresa en la Tabla 1, que presenta la codificación con la cantidad de personas, de experiencias por centros educativos y de las sesiones de EF que contribuyeron a su construcción.

Tabla 1. Fundamentación de los códigos emergentes. Triangulación de las atribuciones emocionales percibidas en el prácticum

\begin{tabular}{|c|c|c|c|c|}
\hline \multirow{2}{*}{ Código } & \multicolumn{2}{|c|}{ NP } & \multirow{2}{*}{ NCE } & \multirow{2}{*}{ NSEF } \\
\hline & Hombre & Mujer & & \\
\hline $\begin{array}{l}\text { Demostración de afecto positivo hacia los } \\
\text { practicantes }\end{array}$ & 4 & 8 & 12 & 17 \\
\hline $\begin{array}{l}\text { Respuesta positiva del alumnado escolar a las } \\
\text { tareas de aprendizaje }\end{array}$ & 5 & 8 & 13 & 16 \\
\hline Contribuir al aprendizaje del alumnado & 3 & 3 & 6 & 11 \\
\hline $\begin{array}{l}\text { Emociones positivas del alumnado en el } \\
\text { aprendizaje }\end{array}$ & 4 & 3 & 7 & 9 \\
\hline Relación de confianza con el alumnado & 2 & 4 & 6 & 7 \\
\hline Finalización de la práctica & 2 & 3 & 5 & 5 \\
\hline Evaluación del aprendizaje & 2 & 3 & 5 & 5 \\
\hline Ausencia de logros en las metas pedagógicas & 1 & 3 & 4 & 4 \\
\hline Comportamiento disruptivo del alumnado & 1 & 3 & 4 & 4 \\
\hline Incertidumbre en torno al contexto pedagógico & 1 & 3 & 4 & 4 \\
\hline El desafío de gestionar la clase & 2 & 2 & 4 & 4 \\
\hline Ambiente recreativo & 0 & 3 & 3 & 4 \\
\hline Participación activa en la clase & 2 & 1 & 3 & 3 \\
\hline Familiarización al alumnado & 1 & 2 & 3 & 3 \\
\hline Total & 30 & 49 & 79 & 96 \\
\hline
\end{tabular}

NP: Número de personas; NCE: Número de centros educativos; NSEF: Número de sesiones de educación física. Fuente: Elaboración propia con base en los resultados de los diarios reflexivos del alumnado universitario participante.

De acuerdo con los criterios de triangulación en el análisis de contenido, fueron eliminados 12 códigos que no cumplían con la fundamentación establecida. 


\section{Resultados}

Los hallazgos de esta investigación, que responde al objetivo de identificar la percepción y atribución emocional del alumnado universitario de EF en su práctica intermedia, se presentan en 2 familias de códigos y 14 códigos. En la Tabla 2, se presenta la cantidad de veces que las emociones fueron percibidas por los estudiantes practicantes y descritas en forma explícita en las narraciones de los diarios reflexivos.

Tabla 2. Distribución de las emociones percibidas durante la práctica intermedia

\begin{tabular}{lcc}
\hline \multicolumn{1}{c}{ Emoción } & Frecuencia & $\%$ \\
\hline Alegría & 71 & 39.2 \\
Seguridad & 30 & 16.5 \\
Nerviosismo & 21 & 11.6 \\
Entusiasmo & 12 & 6.6 \\
Tranquilidad & 10 & 5.5 \\
Diversión & 8 & 4.4 \\
Relajo & 6 & 3.3 \\
Inseguridad & 5 & 2.7 \\
Tristeza & 4 & 2.2 \\
Satisfacción & 4 & 2.2 \\
Frustración & 3 & 1.6 \\
Enfado & 3 & 1.6 \\
Ansiedad & 2 & 1.1 \\
Amor & 2 & 1.1 \\
\hline Total & 181 & 99.6 \\
\hline
\end{tabular}

Fuente: Elaboración propia con base en los resultados de los diarios reflexivos del alumnado universitario participante.

El significado relacional que los estudiantes han señalado para cada emoción se presenta a continuación en el apartado de las familias que representan las atribuciones emocionales.

\subsection{Interacción social}

Esta familia se compone de 8 códigos descritos en la Tabla 3, los cuales han sido identificados por los estudiantes en práctica como causales de sus emociones. Las atribuciones de esta familia, se originan de procesos pedagógicos basados en la comunicación practicante-alumnos/as, alumnos/as-alumnos/as y practicante-tutor. 
Tabla 3. Definición de los códigos de la familia interacción social

\begin{tabular}{|c|c|}
\hline Código & Definición \\
\hline $\begin{array}{l}\text { Demostración de afecto } \\
\text { positivo hacia los practicantes }\end{array}$ & $\begin{array}{l}\text { Abrazos, sonrisas, atención, gestos de cariño, diálogos } \\
\text { amistosos y actitud de aceptación de los escolares y } \\
\text { el profesorado con los estudiantes de pedagogía. AC: } \\
\text { alegría, seguridad, tranquilidad y amor. }\end{array}$ \\
\hline $\begin{array}{l}\text { Respuesta positiva del } \\
\text { alumnado a las tareas de } \\
\text { aprendizaje }\end{array}$ & $\begin{array}{l}\text { Implicación de los escolares en las tareas de } \\
\text { aprendizaje. AC: seguridad, alegría, tranquilidad, } \\
\text { relajo y satisfacción. }\end{array}$ \\
\hline $\begin{array}{l}\text { Emociones positivas del } \\
\text { alumnado en el aprendizaje }\end{array}$ & $\begin{array}{l}\text { Percepción de emociones en los escolares que } \\
\text { favorecen su bienestar subjetivo durante el proceso de } \\
\text { aprendizaje. } \\
\text { AC: alegría, entusiasmo, diversión y tranquilidad. }\end{array}$ \\
\hline $\begin{array}{l}\text { Relación de confianza con el } \\
\text { alumnado }\end{array}$ & $\begin{array}{l}\text { Percepción de los practicantes que les indica que los } \\
\text { escolares se sienten familiarizados con ellos. } \\
\text { AC: seguridad, alegría, diversión y entusiasmo. }\end{array}$ \\
\hline Finalización de la práctica & $\begin{array}{l}\text { Última sesión de la práctica en el centro escolar. } \\
\text { AC: tristeza y alegría. }\end{array}$ \\
\hline $\begin{array}{l}\text { Ausencia de logros en las metas } \\
\text { pedagógicas }\end{array}$ & $\begin{array}{l}\text { Percepción de que las acciones pedagógicas no } \\
\text { cumplieron su objetivo. AC: tristeza, el nerviosismo y } \\
\text { la frustración. }\end{array}$ \\
\hline $\begin{array}{l}\text { Comportamiento disruptivo } \\
\text { del alumnado }\end{array}$ & $\begin{array}{l}\text { Casos de violencia y de indisciplina durante el } \\
\text { aprendizaje. AC: nerviosismo, enfado y frustración. }\end{array}$ \\
\hline Familiarización al alumnado & $\begin{array}{l}\text { Conocer con mayor detalle a los escolares. } \\
\text { AC: alegría y seguridad. }\end{array}$ \\
\hline
\end{tabular}

AC: atribución causal. Fuente: Elaboración propia con base en los resultados de los diarios reflexivos del alumnado universitario participante.

El afecto positivo transmitido a los practicantes es el código más mencionado en las sesiones de EF, el cual es reconocido por provocar emociones positivas para el bienestar subjetivo (BS), como se indica en la siguiente cita: "comencé a sentir el amor y la alegría que poseían los niños, ya que, al llegar a la Escuela, gran cantidad de niños se acercaron y demostraron su cariño por medio de abrazos y pidiendo que juegue con ellos» (E1). Por medio de esta cita se refleja la transferencia emocional que se produce en la comunicación verbal y no verbal. La implicación de los escolares en el aprendizaje es percibido como causal de emociones positivas para el BS, como se señala en la siguiente cita: "en esta clase, en especial, me sentí muy segura y de a poco tomando confianza 
con el curso, el profesor me hizo dirigir la elongación y fue una buena experiencia, ya que todos los niños colaboraron y siguieron mis órdenes» (E3). Cuando los practicantes identifican que el alumnado escolar vivencia emociones positivas para su BS durante el aprendizaje, es comprendido como causal de emociones positivas para el BS, lo cual expresa que las emociones se podrían transferir por medio de la observación e interacciones con una comunicación indirecta, como se indica en la siguiente cita: «senti alegría, porque la clase en general estuvo calmada y divertida para todos, además de que los estudiantes se sentían entusiasmados, lo cual alegra más aun la clase» (E16).

Por medio de la interacción practicante-alumno, los primeros/as pueden percibir que el alumnado escolar se relaciona con una confianza que en principio era distinta, lo cual les desencadena emociones positivas para su BS, como se indica en la siguiente cita: "hoy me sentí con más entusiasmo que otros días, ya que los alumnos me demostraron la confianza que se está creando conmigo preguntando hasta la más leve duda en relación a la clase y a su evaluación» E22). En el último día de práctica, algunos estudiantes de pedagogía en EF sintieron emociones positivas y negativas para su BS. Las primeras son relacionadas al logro de haber concluido el proceso y las segundas, al término de las relaciones sociales que se habían construido con los escolares, como se expresa en la siguiente cita: «estaba triste, ya que sería mi última clase con ellos, y quizás no los vuelva a ver, y les tome cariño (E7). Esta tristeza puede ser valorada como un aspecto positivo a nivel pedagógico, ya que demuestra el vínculo afectivo construido en la práctica, de modo que para que no se convierta en una consecuencia negativa, requiere la aplicación de un adecuado ajuste emocional.

La percepción de fracaso, en las metas pedagógicas, es interpretada como causante de emociones negativas para el BS, sin embargo, podrían ser consideradas positivas a nivel pedagógico, debido a que se producen por un deseo de afectar positivamente la realidad, lo cual refleja un compromiso con el quehacer educativo, como se manifiesta en la siguiente cita representativa: "me sentía frustrada, porque no lograba hacer que escucharan las explicaciones de las actividades» (E10). Una meta pedagógica no necesariamente tiene que estar formulada desde el currículo, debido a que, desde el quehacer docente, se formulan constantemente metas que contienen una intencionalidad pedagógica, las cuales pueden o no pueden responder a temas asociados al currículo obligatorio. El comportamiento disruptivo del alumnado es reconocido como motivo de emociones negativas para el BS, como se manifiesta en la siguiente cita: "me sentí nerviosa por pelea entre alumnos» (E4), lo cual demuestra la importancia de que la formación docente incluya contenidos de esta temática, debido a que la práctica pedagógica demanda la necesidad de autorregular las 
emociones en los episodios conflictivos. Percibir que se ha logrado conocer a los escolares es interpretado como causal de emociones positivas para el BS, lo cual también puede significar un aspecto positivo para el quehacer docente, como se señala en la siguiente cita: «emocionalmente me sentí muy segura, ya que habia conocido más a los niños, sabia sus nombres además de que jugamos» (E18).

\subsection{Rol pedagógico}

Esta familia se compone de 6 códigos descritos en la Tabla 4, los cuales han sido identificados por los estudiantes en práctica como causales de sus emociones. Las atribuciones de esta familia se destacan por la influencia que tiene el tipo de función educativa del practicante en el origen de sus emociones.

Tabla 4. Definición de los códigos de la familia rol pedagógico

Código Definición

Contribuir al aprendizaje Aportar a los escolares durante su aprendizaje. AC: escolar seguridad, alegría, entusiasmo.

Evaluar al alumnado Experimentar junto al tutor docente el proceso de valorar el aprendizaje. AC: alegría, entusiasmo y nerviosismo.

El desafío de gestionar una Visualizar o vivenciar la responsabilidad pedagógica total clase de la sesión. AC: inseguridad y nerviosismo.

Incertidumbre en torno al Desconocimiento por parte de los estudiantes en práctica contexto pedagógico sobre las actitudes y comportamientos de los escolares, sobre el estilo pedagógico del tutor y de las actividades pedagógicas. AC: nerviosismo.

Ambiente recreativo Actividades en la clase de Educación Física que no responden al currículum y tienen un carácter lúdico o de celebración. AC: diversión, relajo, tranquilidad y alegría.

Participar activamente en Intervención de los estudiantes en práctica en alguna la clase parte de la sesión de educación física. AC: alegría.

AC: atribución causal. Fuente: Elaboración propia con base en los resultados de los diarios reflexivos del alumnado universitario participante.

Contribuir a los escolares durante su aprendizaje por medio de las intervenciones pedagógicas es el código más citado de esta familia en las sesiones de EF y es comprendido como causal de emociones positivas para el BS, lo cual se expresa en la siguiente cita: «me sentí más entusiasmada, con ganas de participar más, con más alegría, ya que habia que ir guiando a los niños, habia que ir al lado de ellos diciéndoles qué hacer y, si era necesario, habia que trotar con 
ellos» (E10). Participar del proceso de evaluación del aprendizaje es interpretado como causal de emociones positivas y negativas para el BS. Las emociones positivas se relacionan a la posibilidad de evaluar el aprendizaje por primera vez y a la importancia que representa el proceso. Las emociones negativas son relacionadas a la complejidad del proceso y a la dificultad del alumnado para rendir un test, como se indica en la siguiente cita: «emocionalmente me fue un poco chocante, me dio nerviosismo al encontrarme en la evaluación a tres alumnos (una niña y dos niños), los cuales están evidentemente sobre un peso adecuado a su edad, lo que les impide realizar un buen test» (E22). Proyectar o vivenciar la posibilidad de gestionar la sesión de EF es señalado como causal de emociones negativas para el BS, lo cual puede ser explicado a la ausencia de experiencia en el rubro, como se indica en la siguiente cita: Senti nerviosismo puro, el profesor no asistió al colegio por licencia médica y realicé las clases, aunque pude trabajar de muy buena forma (E15).

El desconocimiento en torno a la conducta de los escolares y el profesorado tutor de la práctica, así como de las actividades o contenidos a desarrollar en las sesiones de EF, es interpretado como un motivo causal de emociones negativas para el BS, como se indica en la siguiente cita: «me sentí con un poco de nervios, porque no sabia a lo que iba, debido a ser la primera clase» (E2). Algunas practicantes señalan que hay días en que la sesión de EF es cedida a otras actividades con fines recreativos, lo que también modifica el rol de la docencia, y es reconocido como un motivo causal de emociones positivas para el BS, como se indica en la siguiente cita: «tranquila y alegre, debido a que la clase se cedió para celebrar el cumpleaños de Pascal» (E4). Estas emociones positivas para el bienestar, desde una perspectiva pedagógica, pueden ser valoradas como negativas, debido a que, a pesar de la buena intención de la actividad, es un problema que la sesión de EF se utilice para actividades no académicas, ya que la cantidad de horas de esta asignatura es muy baja y el alumnado necesita realizar actividad física en forma sistemática. Lograr una participación activa en las sesiones de EF es un reconocido como un motivo de emociones positivas para el BS, como se indica en la siguiente cita: «me sentí muy alegre, debido a que pude participar activamente en la clase realizando algunas partes de la clase» (E12).

\section{Discusión}

La transmisión de emociones que se ha identificado en la interacción social durante el proceso pedagógico es coherente con el descubrimiento de las neuronas espejo o también conocidas como neuronas de la empatía (Morris, 2014). 
Precisamente, este hallazgo comprueba que alumnado escolar «puede hacer propios los gestos, posturas, expresiones y emociones del docente, sucediendo lo mismo al revés, donde el docente puede percibir esas expresiones del alumnado» (Mujica et al., 2018c, p. 121). Por consiguiente, este conocimiento nos revela que las emociones son contagiosas, de modo que tienen una importante influencia en el ánimo o ambiente colectivo del entorno educativo (Mayorga, 2015), por lo tanto, uno de los desafíos del profesorado es tomar conciencia de sus propias emociones y suscitar emociones con un valor positivo para el BS del alumnado y también para el proceso pedagógico.

Entre las atribuciones emocionales, se encuentra el logro o el fracaso de metas pedagógicas, así como la percepción de haber contribuido al aprendizaje de los escolares, lo cual se vincula a la función motivacional que cumplen las emociones, ya que se relacionan de dos formas con esa orientación: la primera es que actúa como un motivo, porque energiza y dirige la conducta, y la segunda sirve como un sistema de indicador continuo para señalar cómo está yendo la adaptación (Reeve, 2010). En este sentido, es muy beneficiosa la actitud optimista del profesorado en la interacción con su alumnado (Béjar, 2014), pero no se debe confundir "con la mera palabrería de pronósticos improbables, sino que debería concretarse en dar énfasis a pequeñas metas cuya consecución motiven más al alumnado» (Mujica et al., 2018c, p. 121). En cuanto al entusiasmo, ha sido percibido en ambas familias de códigos, destacándose como una emoción importante en la motivación profesional (González-Blasco, Moreto, Janaudis, De Benedetto, Delgado-Marroquín y Altisent, 2013), al igual que la seguridad en la motivación durante el desempeño docente (Vidal, García y Pacheco, 2010) y las experiencias placenteras en la motivación intrínseca durante las clases de EF (Moreno-Murcia, Joseph y Huéscar, 2013). Estas relaciones representan uno de los motivos por los que actualmente se considera que las emociones positivas para el BS favorecen el aprendizaje (López, 2016).

La interrelación de las emociones con los razonamientos y las interpretaciones morales genera que las emociones respondan a las teorías implícitas que han sido configuradas en la mente de los futuros docentes, ya sea en los contextos formales o informales de aprendizaje (Mujica-Johnson y Jiménez, 2019), lo cual se reflejó en el proceso de la práctica cuando los participantes le otorgaron un valor correcto o incorrecto al comportamiento de los escolares o también cuando valoraron sus acciones en el aula. Esto no es ajeno a los modelos ideales de didáctica que se les ha enseńado o han aprendido en forma independiente, de igual forma cuando visualizan el centro educativo y sienten nerviosismo ante la incertidumbre o los comentarios negativos de otras 
personas, generándoles emociones positivas o negativas para su BS, lo cual ya ha sido identificado en otros estudios que han investigado un contexto similar (Orellana et al., 2015; Mujica et al., 2015; Mujica, 2018b). Las teorías implícitas, según su significado, pueden favorecer o dificultar el quehacer docente, por lo que una de las formas de contribuir a la formación de profesores es propiciar la reflexión de la propia práctica educativa (Gómez, 2008), para de esta forma comprender las interacciones emocionales que acontecen en los procesos sociales de la escuela (Arriagada, Martínez, Cresp, Arellano y Vargas, 2015).

\section{Conclusiones}

De acuerdo con la conciencia emocional durante la práctica pedagógica, se puede concluir, al asociar las emociones con la clasificación del BS, que el alumnado ha percibido, mayoritariamente, emociones positivas $y$, en bastante menor frecuencia, emociones negativas. Estas emociones son percibidas durante situaciones caracterizadas por la interacción social y por el rol pedagógico. En cuanto al valor educativo de las emociones, la mayoría de las emociones surge por el deseo de querer lograr una adecuada práctica pedagógica y contribuir a la educación del alumnado escolar. Se identificaron emociones negativas para el BS que favorecen el quehacer educativo y emociones positivas que perjudican el desarrollo de la EF, lo cual demuestra la importancia de atender el significado contextual de las emociones.

Con relación a la atribución causal de las emociones positivas para el BS, los estudiantes las asocian en mayor parte a motivos de origen afectivo, lo que manifiesta que estas emociones se reproducen por medio de la comunicación verbal, a través de diálogos amistosos; por medio de la comunicación no verbal, con abrazos, sonrisas o actitud de aceptación; por medio de la comunicación directa, en las relaciones de confianza; y en la comunicación no directa, cuando se observa a distancia que un alumno se encuentra alegre, divertido o entusiasmado durante las tareas de aprendizaje. Estas asociaciones manifiestan la importancia que tiene para el ambiente de aprendizaje la calidad afectiva de la interacción social. Con respecto a la atribución causal de las emociones negativas para el BS, los estudiantes las asocian al término del proceso, lo cual refleja el vínculo afectivo que establecieron en el centro educativo; al fracaso en las metas pedagógicas; al comportamiento disruptivo del alumnado; y a la complejidad que significa asumir el rol pedagógico. Estas emociones cumplen una importante función social, por lo que es de gran necesidad que los futuros docentes desarrollen competencias que les ayuden a conseguir un ajuste emocional. 
A pesar de la formación en la dimensión emocional que recibieron los estudiantes universitarios antes del estudio, hubo participantes que demostraron dificultad para percibir y comprender sus emociones, por lo que se recomienda para futuros estudios, realizar talleres con carácter teórico-práctico orientados a la conciencia emocional. En el mismo sentido, es preciso indicar que entre las limitaciones del estudio se encuentra el carácter interpretativo para explicar la relación de las emociones del alumnado universitario con el entorno pedagógico. Por consiguiente, las asociaciones mencionadas indican una causalidad identificada por una vía esencialmente subjetiva, la cual es coherente para indicar certezas sobre la temática, pero también está sujeta a las limitaciones de la percepción humana. En consecuencia, es fundamental contrastar las interpretaciones de este estudio con otros que utilicen enfoques metodológicos similares como también diferentes.

\section{REFERENCIAS BIBLIOGRÁFICAS}

Aguilar, S. y Barroso, J. (2015). La triangulación de datos como estrategia en investigación educativa. Revista de Medios y Educación, 47, 73-88. https:// doi.org/10.12795/pixelbit.2015.i47.05

Arriagada, C., Martínez, C., Cresp, M., Arellano, R. y Vargas, R. (2015). Formación inicial docente, teorías y paradigmas en educación física. Revista Ciencias de la Actividad Física, 16(2), 37-46.

Béjar, M. (2014). Una mirada sobre la educación. Neuroeducación. Revista Padres y Maestros, 355, 49-52. https://doi.org/10.14422/pym.v0i355.2622

Bisquerra, R. (2005). La educación emocional en la formación del profesorado. Revista Interuniversitaria de Formación del Profesorado, 54, 95-114.

Bisquerra, R. Bisquerra, A., Cabero, M., Filella, G., García, E. López, E., Moreno, C. y Oriol, X. (2016). Educación emocional. Propuesta para educadores y familias. Bilbao: Desclée de Brouwer.

Bogdan, R. y Taylor, S. (2009). Introducción a los métodos cualitativos de investigación. Barcelona: Paidós.

Cabezas, M. (2013). Juicios morales y fronteras biológicas: más allá de la frontera razón/emoción. Arbor, 189(762), a052. https://doi.org/10.3989/ arbor.2013.762n 4003

Crespo, E. (1982). Los procesos de atribución causal. Estudios de Psicología, $12,34-45$.

Crespo, E. y Célio, J. (2014). La atribución de responsabilidad: de la cognición al sujeto. Psicología \& Sociedade, 26(2), 271-279. https://doi.org/10.1590/ S0102-71822014000200004 
Del Valle, A. (1998). Educación de las emociones. Revista Educación, 7(14), 169-198.

Gómez, L. (2008). Las teorías implícitas de los profesores y sus acciones en el aula. Revista Electrónica Sinéctica, 30, 1-14.

González, F. (2009). La significación de Vygotski para la consideración de lo afectivo en la Educación: Las bases para la cuestión de la subjetividad. Revista Electrónica Actualidades Investigativas en Educación, 9, 1-24. https://doi. org/10.15517/aie.v9i4.9519

González-Blasco, P., Moreto, G., Janaudis, M., De Benedetto, M., DelgadoMarroquín, M. y Altisent, R. (2013). Educar las emociones para promover la formación ética. Persona y Bioética, 17(1), 28-48. https://doi. org/10.5294/pebi.2013.17.1.2

González, L, Rivera, E. y Trigueros, C. (2014). La interacción social en el contexto del aula de Educación Física. Revista de Currículum y Formación del Profesorado, 18(2), 305-320.

Jordán, J. y Méndez, J. (2017). Rasgos esenciales de los profesores excelentes en su relación con los alumnos tras el visionado de películas pedagógicamente valiosas. Estudios sobre Educación, 33, 103-126. https://doi. org/10.15581/004.33.103-126

Koole, S. (2009). The psychology of emotion regulation: an integrative review. Cognition and Emotion, 23(1), 4-41. https://doi.org/10.1080/ 02699930802619031

Lago, F., Presa, I., Pérez, J. y Muñiz, J. (2003). Educación socio-afectiva en secundaria. Un programa de orientación y acción tutorial. Madrid: CCS.

Lavega, P., Aráujo, P. y Jaqueira, A. (2013). Teaching motor and emotional competencies in university students. Cultura, Ciencia y Deporte, 8(22), 5-15. https://doi.org/10.12800/ccd.v8i22.219

Lazarus, R. (2000). Estrés y emoción. Manejo e implicaciones en nuestra salud. Bilbao: Editorial Desclée de Brouwer.

López, B. (2016). Aprendizaje emocionante. Neurociencia para el aula. Madrid: Biblioteca Innovación Educativa.

Marina, J. (2005). Precisiones sobre la educación emocional. Revista Interuniversitaria del Profesorado, 54, 27-44.

Martínez, M. (2006). Validez y confiabilidad en la metodología cualitativa. Paradigma, 27(2), 1-20.

Maturana, H. (2001) Emocionesy lenguaje en educación y política. Santiago: Dolmen. Mayorga, L. (2015). Neuroeducación en las aulas de clase. Revista Do-Ciencia, 3, 43- 45 . 
Mejía, J. (2011). Problemas centrales del análisis de datos cualitativos. Revista Latinoamericana de Metodología de la Investigación Social, 1(1), 47-60. Ministerio de Educación. (2013). Formación en sexualidad, afectividad y género. Santiago: Gobierno de Chile.

Ministerio de Educación. (2014). Estándares Orientadores para carreras de Pedagogía en Educación Física. Santiago: Trama Impresores.

Mora, F. (2017). Neuroeducación. Solo se puede aprender aquello que se ama. Madrid: Alianza.

Moreno, A., Trigueros, C. y Rivera, E. (2013). Autoevaluación y emociones en la formación inicial de profesores de educación física. Revista de Estudios Pedagógicos, 39(1), 165-177. https://doi.org/10.4067/ S0718-07052013000100010

Moreno-Murcia, J., Joseph, P. y Huéscar, E. (2013). Cómo aumentar la motivación intrínseca en clases de educación física. E-motion. Revista de Educación, Motricidad e Investigación, 1, 30-39. https://doi.org/10.33776/remo. v0i1.2263

Morris, M. (2014). La neuroeducación en el aula: neuronas espejo y la empatía docente. Revista la vida y la historia, 3(2), 9-18.

Mujica, F. (2018a). Las emociones en la educación física escolar. El aporte de la evaluación cualitativa. EmásF. Revista Digital de Educación Fisica, 51, 64-78.

Mujica, F. (2018b). Percepción de las emociones y su atribución en la formación del docente de Educación Física. Educatio Siglo XXI, 36(3), 397-416. https://doi.org/10.6018/j/350061

Mujica, F., Inostroza, C. y Orellana, N. (2018c). Educar las emociones con un sentido pedagógico: Un aporte a la justicia social. Revista Internacional de Educación para la Justicia Social, 7(2), 113-127. https://doi.org/10.15366/ riejs2018.7.2.007

Mujica-Johnson, F. y Jiménez, A. C. (2019). Percepción emocional en la asignatura de Baloncesto de estudiantes del Grado en Ciencias del Deporte: Estudio Piloto. Cuadernos de Psicología del Deporte, 19(2), 152-166.

Mujica, F., Orellana, N. y Marchant, F. (2015). Atribución emocional en la formación inicial del profesorado de educación física: la práctica educativa. Revista Motricidad Humana, 16(2), 54-60.

Mujica, F. y Orellana, N. (2016). Construcción de la vocación en estudiantes de Pedagogía en Educación Física: un componente subjetivo de la formación profesional. Revista de Educación Física, 34(3), 1-7.

Mujica, F. y Orellana, N. (2018). Autopercepción de la vocación en docentes de educación física escolar en Chile. CPU-e. Revista de Investigación Educativa, 27, 202-229. 
Mujica, F., Orellana, N. y Canepa, P. (2018). Educación emocional en la asignatura de educación física: análisis crítico del valor positivo y negativo de las emociones. Dilemas contemporáneos: Educación, política y valores, 6(1), 1-23.

Orellana, N., Mujica, F. y Luis-Pascual, J. (2015). Atribución causal de las emociones en la formación inicial del docente de educación física. Revista Ibero-Americana de Estudos em Educaçao, 10(4), 1250-1260.

Osses, S., Sánchez, I. y Ibañez, F. (2006). Investigación cualitativa en educación. Hacia la generación de teoría a través del proceso analítico. Estudios Pedagógicos, 32(1), 119-133. https://doi.org/10.4067/ S0718-07052006000100007

Palomera, R., Fernández-Barocal, P. y Brackett, M. (2008). La inteligencia emocional como una competencia básica en la formación inicial de los docentes: algunas evidencias. Electronic Journal of Research in Educational, 6(15), 437-454. https://doi.org/10.25115/ejrep.v6i15.1292

Pérez-López, R., Morales-Sánchez, V., Anguera, M.y Hernández-Mendo, A. (2016). Modelo tridimensional de la calidad en organizaciones deportivas: calidad emocional em usuários/as infantiles. Cuadernos de Psicología del Deporte, 16(1), 143-150. https://doi.org/10.4321/S1578-84232015000100014

Prieto, M. (2018). La psicologización de la educación: implicaciones pedagógicas de la inteligencia emocional y la psicología positiva. Educación XX1, 21(1), 303-320. https://doi.org/10.5944/educxx1.20200

Rapley, T. (2014). Los análisis de la conversación, del discurso y de documentos en investigación cualitativa. Madrid: Morata.

Reeve, J. (2010). Motivación y emoción. Santa Fe: MacGraw-Hill.

Rieffe, C., Oosterveld, P., Miers, A., Meerum, M. y Ly, V. (2008). Emotion awareness and internalizing symptoms in children and adolescents: the emotion awareness questionnaire revised. Personality and Individual Differences, 45, 756-761. https://doi.org/10.1016/j.paid.2008.08.001

Rodríguez-Fernández, A. y Gońi-Grandmontagne, A. (2001). La estructura tridimensional del bienestar subjetivo. Anales de Psicología, 27(2), 327-332.

Rovira, G., López, V., Lagardera, F., Lavega, P. y March, J. (2014). Un viaje de exploración interior: Emociones y estado de ánimo en la práctica motriz introyectiva. Educatio Siglo XXI, 32(1), 105-125. https://doi. org/10.6018/j/194111

Salmurri, F. (2015). Razón y emoción. Recursos para aprender y enseñar a pensar. Barcelona: RBA.

Samper-García, P., Mesurado, B., Richaud, M. y Llorca, A. (2016). Validación del cuestionario de conciencia emocional en adolescentes espańoles. 
Interdisciplinaria, 33(1), 163-176. https://doi.org/10.16888/ interd.2016.33.1.10

Sanz, M., Menéndez, F., Rivero, M. y Conde, M. (2017). Psicología de la motivación. Teorias y prácticas experimentales. Madrid: Sanz y Torres.

Tyng, C., Amin, H., Saad, M. y Malik, A. (2017). The influences of emotion on learning and memory. Frontiers in Psychology, 8, 1454. https://doi. org/10.3389/fpsyg.2017.01454

Um, E., Plass, J., Hayward, E. y Homer, B. (2012). Emotional design in multimedia learning. Journal of Educational Psychology, 104(2), 485-498. https://doi.org/10.1037/a0026609

Vidal, F., García, J. y Pacheco, D. (2010). La motivación en los profesores. International Journal of Developmental and Educational Psychology, 3, 937-942.

Vogel, S. y Schwabe, L. (2016). Learning and memory under stress: implications for the classroom. Science of Learning, 1, 1-1. https://doi.org/10.1038/ npjscilearn.2016.11 